\title{
STUDY ON THE MIXING PHENOMENA OF FILM COOING JET BLOWING THROUGH SHAPED HOLES
}

\author{
Satoshi HADA*, Shohei MORI**, Kenichiro TAKEISHI**, Masaharu KOMIYAMA** \\ and Yutaka ODA** \\ *Mitsubishi Heavy Industries, Ltd, 2-1-1, Shinhama, Araicho, Takasago, Hyogo, 676-8686, Japan \\ **Dept. of Mech. Eng., Osaka University, 2-1, Yamadaoka, Suita, Osaka 565-0871, Japan
}

\begin{abstract}
To understand the mixing phenomena of film cooling air with mainstream is very important to attain higher film cooling performance. This paper describes the detailed mixing mechanism of film cooling air with mainstream using a low-speed wind tunnel. The three different shaped film cooling holes and a conventional circular hole were used in the experiment for comparison. The time-mean and instantaneous fields of velocity and film cooling effectiveness were measured in high resolution by PIV and LIF, respectively. The experimental results show that fine vortex structures are formed in the shear layer between film cooling air and mainstream. Numerical simulation has been also conducted to examine detailed mechanism of the mixing process, using Detached Eddy Simulation (DES). The DES revealed that pre-mixing of coolant jet and mainstream occurs inside a shaped hole (c), which has relatively long and deep diffused exit. This leads to deterioration of film cooling effectiveness at the downstream.
\end{abstract}

Keywords: Gas turbine, Film cooling, Mixing phenomena, PIV, Acetone LIF

\section{INTRODUCTION}

Gas turbines are used for aircraft propulsion and power generation system. These days, in respect of the environment problems such as global warming, gas turbines are needed to increase the thermal efficiency. Increasing the turbine inlet temperature (TIT) plays a critical role in increasing the thermal efficiency and the power output of advanced gas turbines, and with an increase in the TIT, turbine blades have a higher thermal load. In general, gas turbine blades are cooled externally by film cooling. It is achieved by injecting relatively cooler air from the internal coolant passages out of the blade surface in order to form a protective layer between the blade surface and the hot gas-path flow. The interaction between film cooling air and mainstream, which is representative of film cooling, forms a shear layer that initiates the mixing and leads to a decay of the cooling performance along a blade surface. Thus, it is very important to understand the mixing phenomena of film cooling air with mainstream hot gas to attain higher film cooling effectiveness. But the flow through film cooling holes is known to be extremely complicated because it accompanies numerous complicated vortices [1][2].

To understand the complicated mixing phenomena of film cooling, a large number of experimental works have been conducted. A review paper by Goldstein summarized early works in this area [3]. There have been many studies on film cooling flows blowing through inclined discrete holes, including Eriksen and Goldstein [4], Bernsdorf et al. [5]. The efforts to improve film cooling efficiency by adopting film cooling holes with expanded exits have been investigated experimentally by Bell et al. [6] and Yu et al. [7]. Three-dimensional calculations using high spec computers and enhanced turbulence models have been conducted to simulate the behaviour of the detailed flow of film cooling by Medic and Durbin [8] and Guo et al. [9]. Optimization of film cooling hole geometry using numerical analysis has been investigated by Walters and Leylek [10].

Though the time-averaged data is very important, instantaneous flow fields must be measured and analyzed to understand the physics and the mixing mechanism of the coolant flow. Recently, Tokunaga et al. [11] have developed a measuring system to obtain instantaneous fields of film cooling effectiveness using the Laser-Induced Fluorescence (LIF) method, and demonstrated that it can be a strong tool to investigate the mechanism of mixing process in film cooling in view of instantaneous fields.

In this paper, the temperatures distribution are firstly measured for the conventional circular hole and the three different shaped holes, by using an array of K-type thermocouples mounted on a two-axis traversing system [12]. Next, the mixing phenomena of film cooling air with mainstream were investigated in respect of the flow field and film cooling efficiency distribution. Then, the unsteady flow fields are calculated by FLUENT with a detached eddy simulation (DES) as a RANS/LES hybrid model. Lastly, the numerical results were compared with the experimental results, and the prediction capability of the numerical simulation was discussed [13][14].

\section{EXPERIMENTAL METHOD}

\subsection{Experimental Apparatus}

The present experiment has been conducted using a scale-up model of film cooling holes installed on the bottom surface of a low-speed wind tunnel in order to allow detailed probing of flow features. A schematic view of the wind tunnel and the laser light supply system is shown in Figure 1. The wind tunnel has an inlet nozzle with a 9:1 
contraction ratio. The test section is $300 \mathrm{~mm}$ wide, $300 \mathrm{~mm}$ height, and $1950 \mathrm{~mm}$ long. The air speed inside the test section can be varied from 0 to $40 \mathrm{~m} / \mathrm{s}$. For the free-stream velocity of $20 \mathrm{~m} / \mathrm{s}$, flow at the test inlet section shows excellent spatial uniformity, with a free stream turbulence level less than $0.36 \%$. The Reynolds number of this experiment is one tenth to that of the actual equipment. A film cooling hole is located $250 \mathrm{~mm}$ downstream from the entry of the test section.

Figure 2 shows the geometry of the test model with film cooling holes. The model is made of Bakelite. The guide channels to the exits of film cooling holes were inclined at 30 degree toward the main flow direction. The shaped holes are composed of a round tube section with an exit, where a fan-shaped diffuser exists with 15-degree divergence angles on the both lateral sides. The shaped hole (a) is the base geometric form. The shaped hole (b) has no diffused angle in the flow direction. The shaped hole (c) has the flow channel expanded from the deeper point than the shaped hole (a). The diameter of the guide channel was 5 $\mathrm{mm}$. Coordinate axes and the origins were also shown in Figure 2. Blowing ratio, which is defined as Eq.(1), was controlled by changing the film cooling flow rate in this

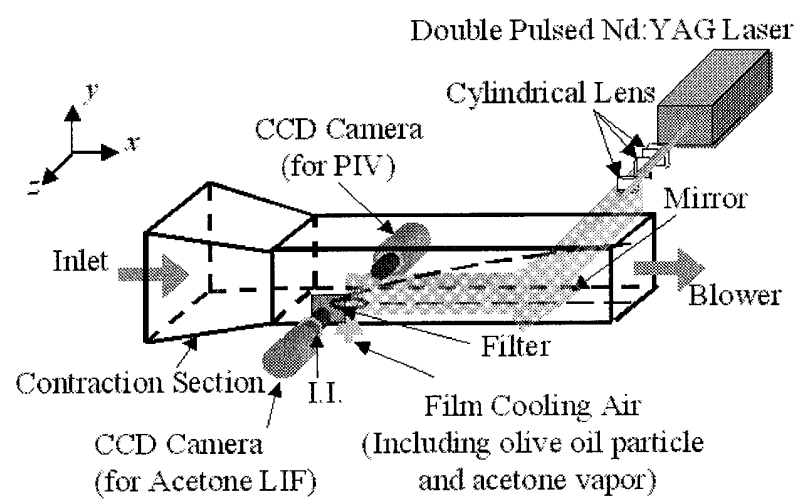

Fig.1 Experimental Apparatus circular hole

shaped hole (b)
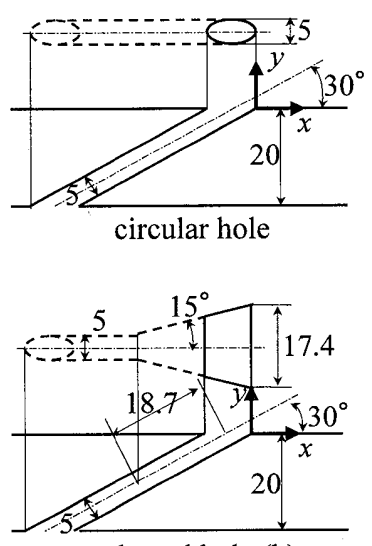
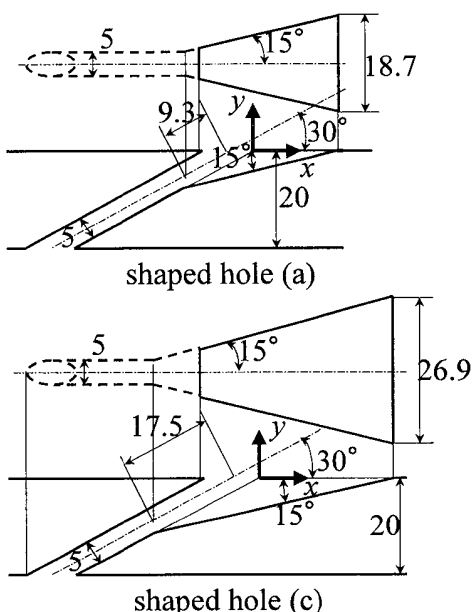

shaped hole (c)
Fig. 2 Film Cooling Hole Models

Table 1 Experimental Conditions

\begin{tabular}{|l|c|}
\hline Mainstream velocity $u_{\infty}[\mathrm{m} / \mathrm{s}]$ & 20 \\
\hline Blowing ratio $\rho_{\mathrm{c}} u_{\mathrm{c}} / \rho_{\infty} u_{\infty}$ & $0.5,1.0,1.5$ \\
\hline Film cooling diameter $d[\mathrm{~mm}]$ & 5 \\
\hline Turbulence intensity $T u[\%]$ & 0.5 \\
\hline Boundary layer thickness $\delta[\mathrm{mm}]$ & 25 \\
\hline
\end{tabular}

study.

$$
M=\frac{\rho_{c} u_{c}}{\rho_{\infty} u_{\infty}}
$$

The temperature distributions were measured by using an array of K-type thermocouples mounted on a two-axis traversing system. In this study, film cooling effectiveness was defined by the mainstream temperature $T_{\infty}$, the coolant temperature $T_{c}$, and the local air temperature $T_{f}$ as follows.

$$
\eta=\frac{T_{\infty}-T_{f}}{T_{\infty}-T_{c}}
$$

The temperature of free-stream air in the channel and film cooling air from the hole were about $15^{\circ} \mathrm{C}$ and $60^{\circ} \mathrm{C}$, respectively. Table 1 shows experimental conditions.

\subsection{Particle Image Velocimetry (PIV)}

In this study, PIV was used to capture instantaneous velocity fields. The secondary flow blown through film cooling holes contains fine particles of olive oil as a tracer, which have $1 \mu \mathrm{m}$ in diameter. The particles of olive oil were generated by bubbling the compressed air into a pool of olive oil through a Raskin nozzle in a pressure vessel. In the vessel, air bubbles burst at the olive-oil/air interface, and then $1 \mu \mathrm{m}$ olive oil particles were released in the air. A dual-pulsed Nd:YAG laser, the wavelength of $532 \mathrm{~nm}$, was employed to illuminate the tracers. Three cylindrical lenses were used to form a laser sheet. The laser sheet was guided by a mirror located downstream and illuminated the test window as shown in Figure 1. The particle pattern formed by the light reflected on the particle surfaces were taken with a CCD camera located at the side of test section and the images were digitally stored on hard disks using the acquisition software HIPIC 8.0. To reduce the effect of reflection from the bottom surface, a background image for each pulse is subtracted from each frame to eliminate the effect of laser light reflection. The pairs of captured images were processed by a recursive local-correlation method to obtain velocity vector fields.

The recursive cross correlation algorithm is realized by repeating the cross correlation calculation from large to small interrogation areas at three levels. This process allows one to reduce the computational load darastically and raises the spatial resolution in the final results. Firstly, a 1st-level sub region is selected and a cross correlation calculation is carried out in the same way a normal correlation processing is conducted. At a next level, using the information at a former level to direct the search window, the correlation process is carried out with a smaller interrogation area, which is downsized by one-quarter from the former level. That is, the previous correlation is used as an estimation to limit the search window for the current correlation. In this way, the probability of locking onto the correct displacement is maintained even at a higher level despite the small data sets. In this study, the smallest interrogation window was $8 \times 8$ pixels with an overlap of $50 \%$.

The PIV measurements were conducted in a plane of symmetry, which is normal to the bottom and parallel to the side walls. Images of tracer particles illuminated by the laser were captured with a CCD camera $(1024 \times 1024$ pixels $)$ above the film cooling holes. The measurement field of the camera was $40 \mathrm{~mm} \times 40 \mathrm{~mm}$. The time interval of each 
double-pulsed laser shots was $10 \mu \mathrm{s}$. The corresponding displacement of a seeding particle between the two flames is about 5 to 6 pixels with the free stream velocity of $20 \mathrm{~m} / \mathrm{s}$. The average flow field was obtained by averaging and processing 500 frame pairs per measurement.

\subsection{Laser Induced Fluorescence (LIF)}

LIF is a measurement method for mass concentration in gaseous flows. The advantages of LIF consist in being non-intrusive, instantaneous, and having high intensity of the fluorescence. In addition, LIF can realize high spatial resolution for the tracer concentration field. The flow is illuminated by a laser sheet whose wavelength is tuned to excite a specific absorption transition of a molecular tracer, which is added for this purpose. A fraction of the molecules in the lower energy level absorbs the incident light and is excited to a higher energy state. When the excited state returns to the states with lower energy, fluorescence light emitted with a different wavelength from the incident light. Thus, the fluorescence light from the tracer is extracted easily from the scattered light by an interference filter.

If the thermal diffusivity of air and the mass diffusivity of the tracer are close, mass transfer can replace the thermal diffusion based on heat/mass transfer analogy. Thus, local film cooling effectiveness $\eta$ can be replaced by Eq. (3).

$$
\eta=\frac{C_{\infty}-C_{f}}{C_{\infty}-C_{c}}
$$

Acetone is known as one of the tracers for concentration measurements in gaseous flows by LIF [15], and the ratio between acetone vapor's mass diffusion coefficient against air $D$ and thermal diffusivity of standard air $\alpha$ is about one to two $\left(D=11.2 \mathrm{~mm}^{2} / \mathrm{s}, \alpha=22.3 \mathrm{~mm}^{2} / \mathrm{s}\right)$. It is enough to apply heat/mass transfer analogy. In addition, acetone LIF is known to show good linearity with respect to partial pressure of acetone in the atmospheric pressure gas.

The Nd:YAG laser at $266 \mathrm{~nm}$ was used as an excitation laser light. The beam was expanded to a sheet of $25 \mathrm{~mm}$ width and $1 \mathrm{~mm}$ thick with four cylindrical lenses, passing the test section as shown in Fig. 1. The saturated acetone vapor in air was produced by bubbling the air in the bottles containing the acetone liquid. In order to obtain optimal acetone vapor concentration, the dry air is mixed with to the saturated air with acetone vapor.

\section{NUMERICAL PROCEDURE}

In this study, Detached Eddy Simulation (DES), which is a RANS/LES hybrid simulation, was applied to simulate the film cooling flow field in the experiments for the shaped film cooling holes (b) and (c). An overview of the symmetric half of the computational domain is shown in Fig. 3 with numerical grids. The domain includes a plenum chamber, a coolant hole and a mainstream region, where the plenum chamber is 120,125 and $43.75 \mathrm{~mm}$ in $z, x$ and $y$ directions and the mainstream region is 120,250 and 75 $\mathrm{mm}$ in $z, x$ and $y$ directions, respectively. A close-up of the grid for each shaped hole is shown in Fig 4. Multi-block structured grid is basically used except for the inside of the shaped hole (c), where unstructured grids were applied to avoid the high grid skewness. The total number of cells for the cases of shaped holes (b) and (c) are 920,000 and $1,600,000$, respectively. All simulations were performed

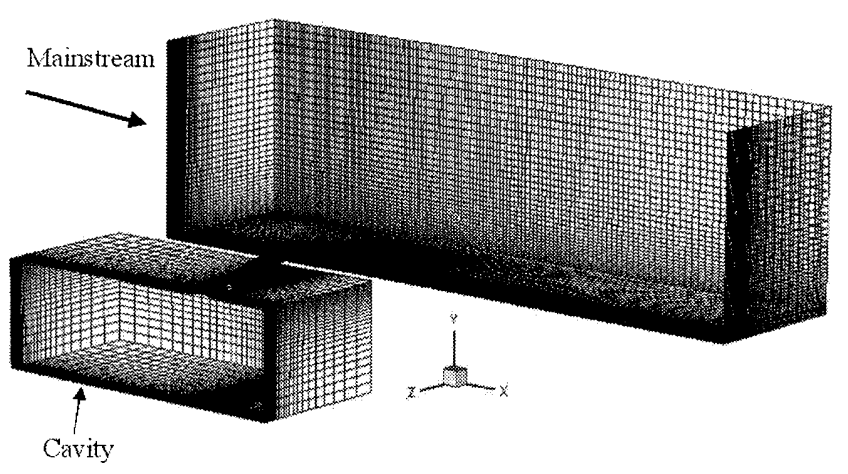

Fig. 3 Computational Grid (Three Dimensional View)

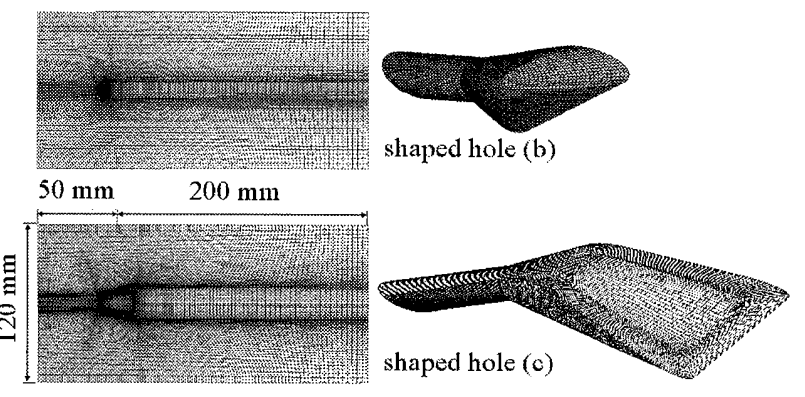

Fig. 4 Computational Grid for Shaped Film Cooling Hole (b) and (c)

using the FLUENT 6.3 with the SIMPLE algorithm. A bounded central differencing scheme, which is a blended scheme of the central difference, 2nd-order upwind scheme, and 1st-order upwind scheme, was applied to discretize the convection terms of momentum equation.

In the present DES, a Spalart-Allmaras (SA) model acts as a one-equation model for the SGS viscosity in LES part by switching the length scale of the model, depending on the distance from the wall and cell spacings. For the inflow condition for the mainstream, profiles of time-mean velocity and modified eddy viscosity for SA model with a uniform temperature of $300 \mathrm{~K}$ were given by the preliminary 2-D wind tunnel simulations, to simulate the boundary layer thickness of $\delta=25 \mathrm{~mm}$ in the experiments. For the inflow condition at the bottom of plenum chamber, an appropriate uniform velocity and temperature of $330 \mathrm{~K}$ was given to realize specified blowing ratio $M$. At the side boundaries, symmetric conditions were applied. For thermal boundary conditions, adiabatic conditions were applied to all wall boundaries. The time step $\Delta t$ was $5 \times 10^{-5} \mathrm{~s}$ and time-mean statics was obtained by the integration over 2000 time steps.

\section{RESULTS AND DISCUSSION}

\subsection{Film Cooling Effectiveness Measured by Thermocouples}

Figure 5 shows that the streamwise distribution of spatial film cooling effectiveness near the bottom surface, which is averaged in the spanwise direction over $-3<z / d<$ 3 at $1 \mathrm{~mm}$ intervals in $x$, for circular and shaped holes.

This figure indicates that the shaped hole (b) shows the best performance on film cooling effectiveness among the four, and the second one is the shaped hole (c). These results seems to be caused by the difference in the geometry of film cooling hole, but no information on the flow fields 


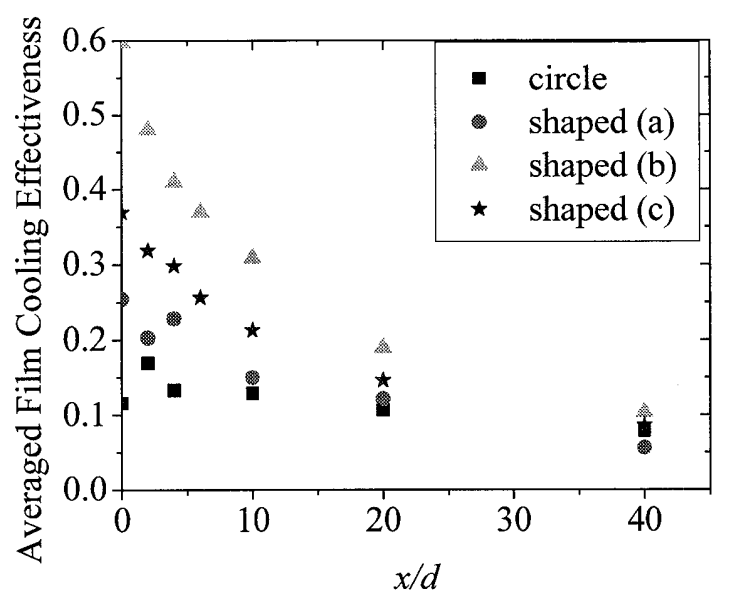

Fig. 5 Film Cooling Effectiveness

(Spanwise Average over $z / d=-3 \sim 3, M=1.0$ )
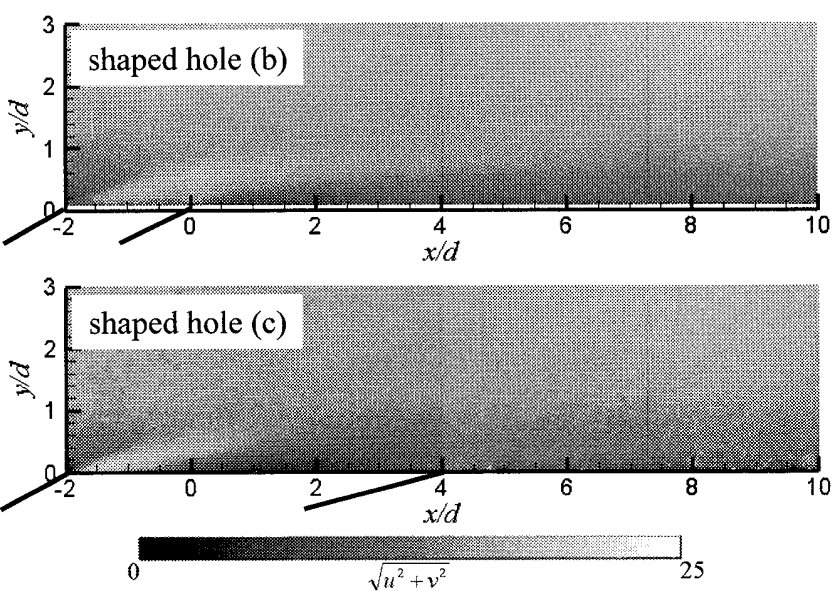

Fig. 6 Mean Velocity Field $(z / d=0, M=1.0)$

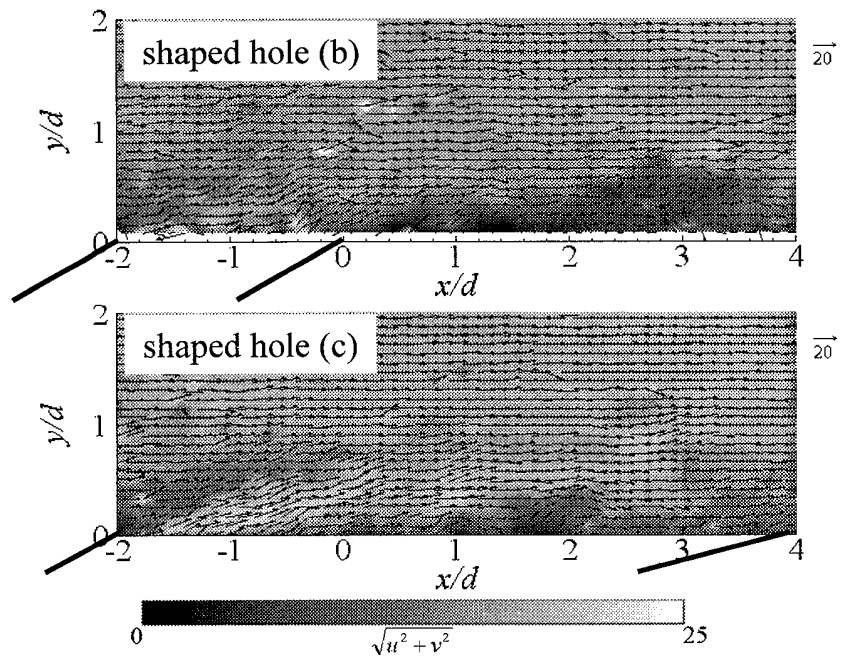

Fig. 7 Instantaneous Velocity Distribution $(z / d=0, M=1.0)$

was obtained near the film cooling hole by the present thermocouple measurement. Therefore, in the following section, the detailed flow field of shaped holes (b) and (c) is discussed using the experimental results of PIV and LIF.

\subsection{Time-Averaged and Instantaneous Velocity Fields by PIV Method}

Figure 6 shows the time-averaged velocity fields obtained by PIV near the exit of shaped holes (b) and (c), respectively, for blowing ratios of $M=1.0$. The results are acquired in two measurement areas, $x / d=-2 \sim 4$ and $4 \sim 10$, and the two images are combined. In the figures, the color represents the velocity magnitude, which is given by $\sqrt{u^{2}+v^{2}}$. Two inclined lines placed below the $x$-axis denote the upstream and downstream edges of the holes. In the case of the shaped hole (b), injected air has the large velocity magnitude over the entire exit of the hole. But, in the case of the shaped hole (c), injected air is concentrated at the upstream-side of the hole as shown by the large velocity magnitude at $-2<x / d<-1$, while having a small velocity magnitude at the downstream-side of the hole exit.

Figure 7 shows instantaneous velocity fields for the shaped holes (b) and (c). A strong shear layer is formed from the upstream edge of film cooling holes by the coolant jet ejected into the mainstream. This shear layer is considered to initiate the Kelvin-Helmholtz instability and enhances the mixing of film cooling air with mainstream. The vortices generated in the shear layer grow up and merge with each other, and eventually produce the large scale fluctuation of the core region of the coolant jet. In addition, velocity vectors in the film cooling flows seem to be disturbed at the hole exit. This disturbed flow seems to originate from flows inside the guide channel, where turbulent separated flows and flow recirculation are often observed near the junction with the plenum chamber.

\subsection{Film Cooling Effectiveness by Acetone LIF Method}

Figures 8 and 9 show the time-averaged film cooling effectiveness distribution obtained by acetone LIF near the exit of shaped holes (b) and (c), respectively, for blowing ratios of $M=0.5,1.0$, and 1.5 . The increase of blowing ratio leads to a thicker layer of film cooling flow for all cases. For the shaped hole (b), the high film cooling effectiveness area is clearly increased near the hole exit as $M$ increases, while it cannot be said for the shaped hole (c). This is probably because the shaped hole (c) has an exit diffused not only in the spanwise direction but also in the streamwise direction, which then results in a larger pressure recovery and a slower velocity at the exit. As confirmed later by numerical simulations, the slower velocity and the deeper hole lead to the inrush of mainstream inside the hole, and thus the pre-mix of coolant jet and mainstream occurs in the hole before the injection. This leads to decrease in film cooling effectiveness at the hole exit.

Next, we shall discuss the profiles of film cooling effectiveness at the cross-sections of $x / d=0,2,4$, and 6 in Fig. 10. Figure 10 shows that the peak positions of film cooling effectiveness are different between the shaped holes (b) and (c). The film cooling effectiveness of the shaped hole (c) has its peak just on the bottom wall, while the jet for the shaped hole (b) has the peak at about $y / d=0.5$. This is caused by the no expansion of the shaped hole (b) in the streamwise direction, which leads to larger velocity at the hole exit. Thus, the coolant jet has enough momentum to penetrate into the mainstream. Figure 10 also shows that the thickness of the coolant jet for shaped hole (b) is larger than that for shaped hole (c).

Figure 11 shows the distribution of the film cooling effectiveness in the instantaneous field. In both film cooling holes, the interface between film cooling jet and 

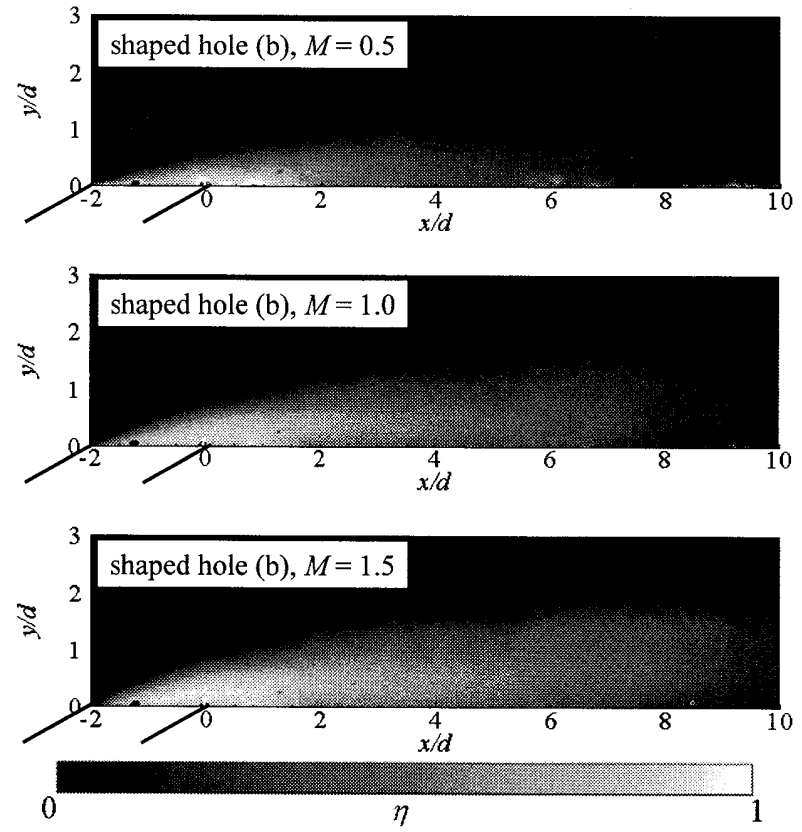

Fig.8 Film Cooling Effectiveness Distribution for Shaped Hole (b)
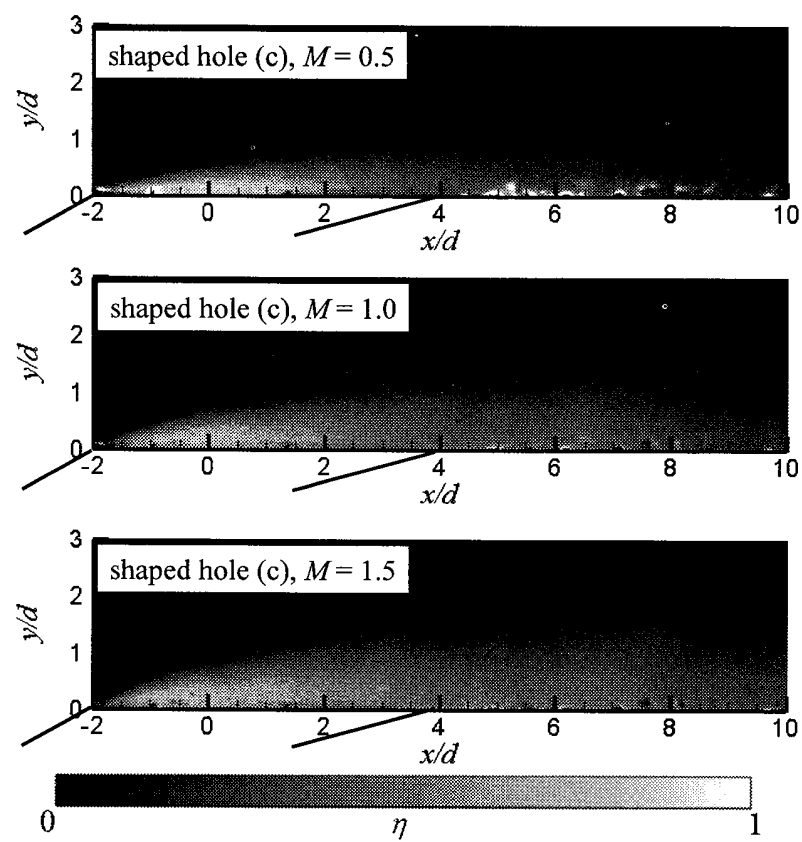

Fig.9 Film cooling Effectiveness Distribution for Shaped Hole (c)

mainstream shows a complex shape in the instantaneous field. The vortex induced by the shear layer forms the complex interface. It can be seen that the vortices are combined with each other and grow gradually toward downstream. In addition, the results of instantaneous flow fields capture the moment when the some parts of coolant air are isolated from the film cooling jet. Such diffusion process of the coolant jet is done by large-scale turbulent eddies in the shear layer. As shown in the figure, film cooling jet is then finally dissipated and disappears at downstream. Since the generation of turbulent eddies in the shear layer originates from the instability of the shear layer growing from the upstream-edge of cooling hole, it is important to examine the effect of the turbulence intensity in the mainstream on the film cooling flow field.
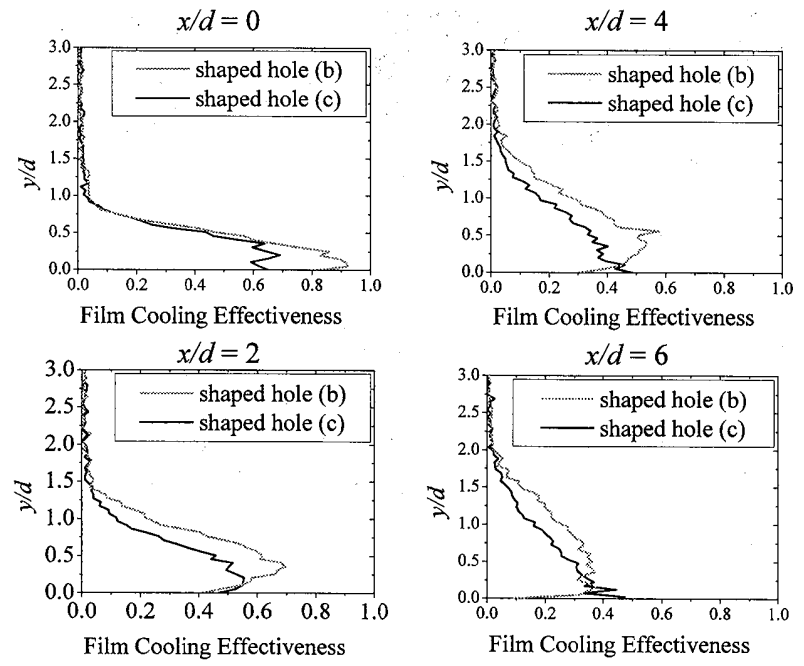

Fig. 10 Line Plot of the Film Cooling Effectiveness for $x / d$ $=0,2,4$ and 6

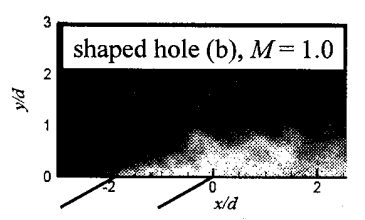

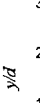

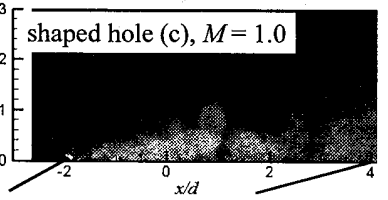

Fig. 11 Film cooling effectiveness in instantaneous fields for shaped hole (b) and (c)

\subsection{Numerical Results (Simulated by DES model)}

Figure 12 shows the comparison of time-mean film cooling effectiveness between DES and LIF at the blowing ratio $M=0.5$ for the shaped hole (b). As the figure indicates, DES prediction shows a longer coverage of coolant than LIF. That is, the coolant flows in DES is less diffusive than in the experiments. This is mainly because the present DES does not have a function to give fluctuating velocities at the inlet boundary, which plays a key role to initiate the flow instability in the shear layer between the coolant jet and mainstream.

This can be confirmed by Fig. 13, showing instantaneous fields of film cooling effectiveness predicted by DES. In Fig. 13, the shape of interface between coolant jet and mainstream looks like smooth in contrast to the fine and complex structures observed in the LIF measurement.

As mentioned earlier, instantaneous fields by DES in Fig. 13 give us an insight into why the shaped hole (c) shows relatively smaller film effectiveness at the exit of the holes when $M$ increases, as shown in Fig. 9. From Fig. 13, it is found that the pre-mixing of coolant and mainstream occurs inside the shaped hole (c) by the inrush of the mainstream due to the longer and deeper shape of the hole compared to the shaped hole (b). Because this phenomenon occurs frequently, the mixing of film cooling air with mainstream is promoted in a statistical sense. As a result, film cooling effectiveness for shaped hole (c) is estimated to be lower than that for shaped hole (b) even for the downstream region. 

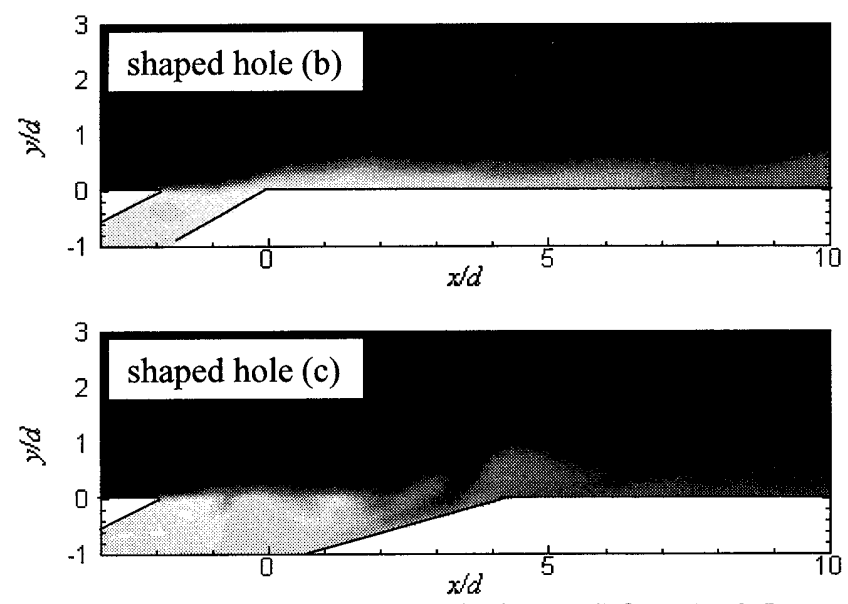

Fig. 13 Instantaneous Results by DES for $M=0.5$
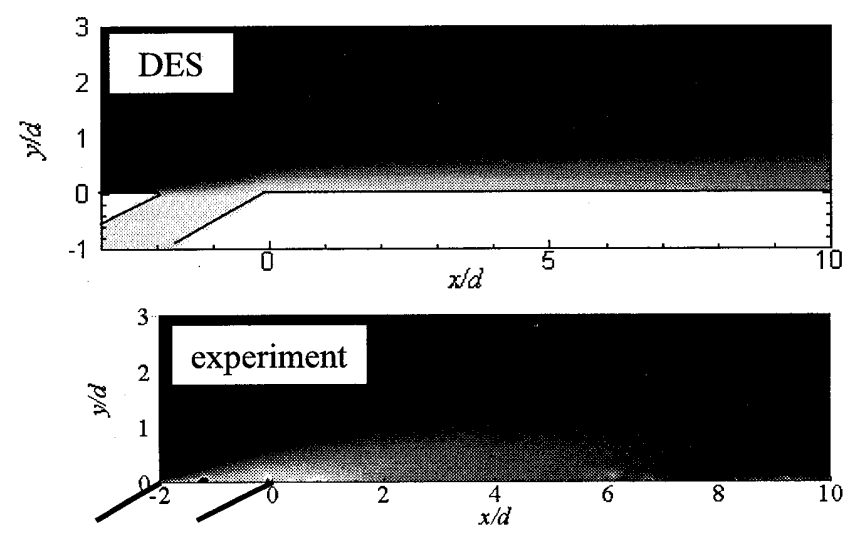

Fig. 12 Film Cooing Effectiveness, Comparison between

DES and LIF for Shaped Hole (b) at $z / d=0$ for $M=0.5$

\section{CONCLUSION}

In this paper, the mixing flow fields of film cooling air with mainstream have been investigated by using thermocouple array, PIV, and acetone LIF for a circular hole and three different shaped holes. As a result, by using acetone LIF and PIV measurement, detailed time-mean and instantaneous spatial profile of film effectiveness and velocity field were obtained for the shaped holes (b) and (c) in high resolution. Numerical analysis by DES has also been conducted to solve the unsteady behavior of film cooling air mixing for the shaped holes (b) and (c). Through these experiments and numerical simulations, the following conclusions were obtained.

1. Shaped holes (b) and (c) show the best and the second best performance, respectively, among the four film cooling holes by thermocouple measurements.

2. Formation of the fine-scale interface between the coolant jet and the mainstream was clearly visualized in the shear layer.

3. Relatively low film effectiveness in the shaped hole (c) than (b) at the hole exit for increased blowing ratio was found to come from the pre-mixing of the coolant jet and mainstream inside the shaped hole by DES.

\section{Acknowledgements}

This study for the $1700^{\circ} \mathrm{C}$ gas turbine is supported by "Ministry of Economy, Trade and Industry" and "Agency for Natural Resources and Energy." The authors wish to express their gratitude to Mitsubishi Heavy Industries, Ltd.

\section{NOMENCLATURE}

$d \quad$ film cooling hole diameter [m]

$u \quad$ cooling jet streamwise velocity $[\mathrm{m} / \mathrm{s}]$

$v \quad$ cooling jet vertical velocity $[\mathrm{m} / \mathrm{s}]$

Subscripts

c coolant

f spatially local film cooling

$\infty \quad$ mainstream flow

\section{REFERENCES}

1. Bunker, R. S., A Review of Shaped Hole Turbine Film-Cooling Technology "Technology Review," ASME J. of Heat Transfer, 127 (2005), pp. 441-453.

2. Bogard, D. G. and Thole, K. A., Gas Turbine Film Cooling, J. of Propulsion and Power, 22 (2006), pp.249-270.

3. Goldstein, R. J., Film Cooling, Advances in Heat Transfer, 7 (1971), pp. 321-379.

4. Eriksen, V, L., and Goldstein, R, J., Heat Transfer and Film Cooling Following Injection Through Inclined Circular Tubes, ASME J. of Heat Transfer, 96 (1974), pp. 239-245.

5. Bernsdorf, S., Rose, M. G. and Abhari, R. S., Modeling of Film Cooling - Part I: Experimental Study of Flow Structure, ASME Turbo Expo 2005, (2005), Paper No. GT2005-68783.

6. Bell, C. M., Hamakawa, H. and Ligrani, P. M., Film Cooling From Shaped Holes, ASME J. of Turbomachinery, 122 (2000), pp. 224-232.

7. Yu, Y, Yen, C. -H. Shin, T. I. -P. and Chyu, M. K., Film Cooling Effectiveness and Heat Transfer Coefficient Distribution Around Diffusion Shaped Holes, ASME J. of Heat Transfer, 124 (2000), pp. 820-827.

8. Medic, G. and Durbin, P. A., Toward Improved Film Cooling Prediction, ASME Journal of Turbomachinery, 124 (2002), pp. 193-199.

9. Guo, X., W, Schröder, W., and Meinke, M., Large-eddy simulations of film cooling flows, Computers \& Fluids, 35 (2006), pp. 587-606.

10. Walters, D, K., and Leylek, J, H., A Detailed Analysis of Film-Cooling Physics: Part I - Streamwise Injection With Cylondrical Holes, ASME J. of Turbomach., 122 (2000), pp 102-112.

11. Tokunaga, D., Takeishi, K., Kumagai, T., and Komiyama, M., Flow Field Measurement for Film Cooling Air by Laser-induced Fluorescence, Proc. of ACGT, (2005), ACGT 2005-086.

12. Sinha, A. K., Bogard, D. G. and Crawford, M. E., Film-Cooling Effectiveness Downstream of a Single Row of Holes With Variable Density Ratio, ASME J. of Turbomach., 113 (1991), pp.442-449.

13. Mizukami, S., Teramoto, S. and Nagashima, T., Numerical Study on the Three-dimensional Flow Structure around a Film Cooling Hole, Proc. of Int. Gas Turbine Congress 2007 (IGTC2007), TS-111, (2007).

14. Tyagi, M. and Acharya, S, Large Eddy Simulation of Film Cooling Flow From an Inclined Cylindrical Jet, ASME J. of Turbomach., 125 (2003), pp.734-742.

15. Lozano, A., Yip, B. and Hanson, R. K., Acetone : a tracer for concentration measurements in gaseous flows by planar laser-induced fluorescence, Experimental in Fluid 13 (1992), pp. 369-376. 